D. OBSERVED STELLAR FLUXES 


\title{
REVIEW OF ULTRAVIOLET AND VISUAL CONTINUUM OBSERVATIONS AND COMPARISONS WITH MODELS
}

\author{
R. C. BLESS \\ Space Astronomy Laboratory, Washburn Observatory, \\ University of Wisconsin, Madison, Wis., U.S.A.
}

\begin{abstract}
This paper first briefly describes model atmosphere grids now available for comparison with observations. The recent recalibration of the absolute energy distribution of $\alpha$ Lyr substantially improves the agreement of models and observations in the visual. Temperature scales determined by various methods agree reasonably well except for the hottest stars. Recent ultraviolet results suggest that earlier observations of $\mathrm{O}$ - and B-type stars indicating large flux deficiencies were probably in error. However, late B- and A-type stars may emit less energy in the UV than that predicted by models which do not include the opacities caused by silicon, magnesium, and carbon.
\end{abstract}

\section{Model Stellar Atmospheres}

Before comparing the observed continua of stars with model atmospheres, it is appropriate first of all to discuss briefly the present state of model atmosphere calculations.

A few years ago a plateau was reached in the computation of standard, LTE models with continuous opacity sources. Mihalas (1964), Strom and Avrett (1965), and Underhill (1963) computed large grids of models in radiative equilibrium, generally with opacities due to bound-free transitions in $\mathrm{H}_{1} \mathrm{H}^{-}, \mathrm{H}_{2}^{+}, \mathrm{He}, \mathrm{He}$ II, free-free transitions, and electron and Rayleigh scattering. Since that time the problem has been to ensure that all of the important sources of continuous opacity have been included, to take into account the effects of the absorption lines, of convection and of rotation and to evaluate the effects of departures from LTE.

The most extensive grids of models which explicitly include absorption lines in the opacities have been calculated by Mihalas (1966), by Mihalas and Morton (1965), by Morton and his students (see, e.g., Hickok and Morton, 1968), and by Klinglesmith and Fischel (1969). Mihalas included the Balmer lines of hydrogen through $\mathrm{H} 20$ in a grid with effective temperatures from $7200 \mathrm{~K}$ to $12600 \mathrm{~K}$ and with $\log g=2,3$, and 4 . In this temperature range the Balmer lines are very strong and are located where much of the flux emerges. They are therefore expected to be the main source of line opacity. At higher temperatures the lines most strongly affecting the structure of the atmosphere are those located farther in the ultraviolet, where most of the star's radiation is emitted. In their calculations of hotter models, the Princeton group included approximately one hundred lines that were expected to have equivalent widths of $2 \AA$ or greater. These lines lie shortward of $1600 \AA$. An approximate expression by Griem $(1960,1962)$ was used for the broadening in the hydrogen Lyman lines while for the other lines a Doppler-damping profile was taken with the damping constant assumed to be 10 times the classical value. Models appropriate to main sequence and nearmain sequence stars with effective temperatures from $14400 \mathrm{~K}$ to $37500 \mathrm{~K}$ have now 
been computed in this way. The effect of the line blanketing is to redistribute the flux from the lines to the regions between the lines and to longer wavelengths extending into the visual spectrum. Thus at B0 the visual spectrum looks like that from an unblanketed model with an effective temperature some $1500 \mathrm{~K}$ higher.

A few attempts have been made to estimate the blanketing effect in the 2000 to $3000 \AA$ region. Here there are not many strong lines and the opacity will apparently be produced by a large number of relatively weak lines rather than by a few strong lines plus weak lines. Hence the calculations are somewhat more difficult and more statistical in nature. Calculations by Elst (1967) show that at type B1 maximum blanketing for this region is reached near 1900 to $2300 \AA$ where it is slightly more than 0.1 magnitudes per $100 \AA$. At longer wavelengths blanketing effects are even smaller.

Another large model atmosphere program has been constructed by Klinglesmith and Fischel at Goddard Space Flight Center. In addition to the usual opacities their program includes the first 40 lines of the Balmer and Lyman series of hydrogen. They will soon publish a Goddard Space Flight Center Note consisting of data for 120 models, including many which are hydrogen deficient. At the Smithsonian Astrophysical Observatory, Strom, Gingerich, and their colleagues have developed model atmosphere programs capable of representing stars over a large portion of the HR diagram. This grid will be published in the Proceedings of the Third Harvard-Smithsonian Atmospheres Conference. Thus it is now possible to compute reasonably sophisticated model atmospheres for stars of spectral type $F$ and earlier, on and near the main sequence, in which at least some of the effects of line opacities are included.

Recently Gingerich, Strom, and others at the Smithsonian Astrophysical Observatory have recognized that the photoionization continua of magnesium, carbon, and silicon can in certain situations be important sources of opacity, and that possibly the wing of Lyman $\alpha$ may affect the structure of the atmosphere throughout the ultraviolet. Our understanding of this last source of opacity seems to be somewhat confused at this point, however. It is apparent that carbon is important and that the ${ }^{3} P$ ground state photoionization limit of silicon at $1520 \AA$ and the corresponding limit from the ${ }^{1} D$ first excited state at $1677 \AA$ cause considerable amount of energy to be redistributed at longer wavelengths up to the Balmer limit. An overabundance of $\mathrm{Si}$ is apparently the cause of the abnormally small Balmer jump observed in Sirius. A few years ago Mihalas (1965) computed two models appropriate to late A- or early F-type stars in which he included convection by means of mixing-length theory. Compared to purely radiative models, the convective models showed a substantial flux deficit shortward of about $1800 \AA$. This deficit increased with greater convective efficiency. It was hoped that observations in the ultraviolet would provide a test of these convection models. However, the silicon opacity in these stars may be large enough even with normal abundance to modify the atmospheric structure in such a way as to vitiate these convective models. Thus the observations may yield information on abundance rather than convection.

Recent calculations by Auer and Mihalas (1969) in which the first three levels of the hydrogen atom are permitted to depart from LTE (detailed balancing is not assumed 
as has been the case in the past) indicate no appreciable change in either the Paschen or Balmer continua compared to the LTE models. This result agrees with observations by Hayes (1967) who found no departures from the LTE values for the ratio of the Balmer and Paschen discontinuities. However, these calculations do show that for the non-LTE model the Lyman continuum is depressed by approximately a factor of 4 at $T_{\mathrm{e}}=15000 \mathrm{~K}$ and by a factor of 2 at $T_{\mathrm{e}}=25000 \mathrm{~K}$. This result could affect considerations of the excitation of gaseous nebulae and in particular the effective temperatures of the exciting stars derived from observations of the nebular flux.

\section{Visual Observations}

On the observational side, a new spectrophotometric calibration of early-type stars by Hayes (1967) has considerably improved the comparison of continuous energy distributions with predictions of model atmospheres. Figure 1 compares the absolute calibrations of Hayes (1967), Code (1960), and Oke (1964). Oke's was not an absolute calibration in the usual sense; rather he used an unblanketed model computed by Mihalas $\left(T_{\mathrm{e}}=9500^{\circ}, \log g=4.44\right)$, which best represented the data on Vega known in 1964, as an interpolative device. Code's calibration was a composite one, including observations made by several investigators, principally Kienle and his co-workers, Williams, and Hall (see Code, 1960), as well as observations by Whitford and Code in the red. The calibration he adopted was a mean of these measurements and agrees well with the recent measurements of Hayes except in the region from about $5000 \AA$ shortward to the Balmer jump. In this region Kienle's observations were considerably higher than those of Williams, and apparently better represented the continuum of

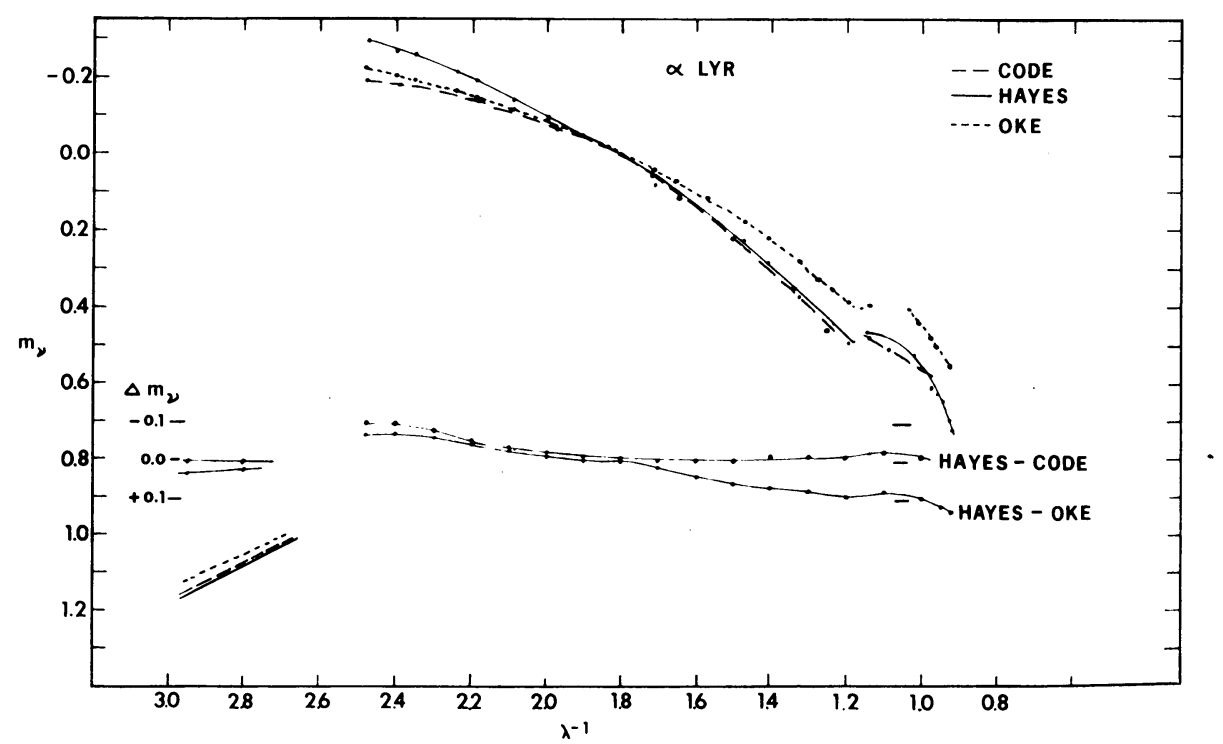

Fig. 1. Absolute calibration of Vega. 
Vega than did the mean Code drew between the two sets of observations. That the Balmer jump should be greater than that given by Code was also indicated by measurements of Bahner (1963), who found good agreement with Code's energy distribution, except for the Balmer jump, which Bahner increased by about $0^{\mathrm{m}} .13$. With Code's calibration one cannot fit a model atmosphere to the Paschen continuum both shortward and longward of about $5500 \AA$. With Oke's energy curve one can fit the Paschen continuum, but the observed Balmer discontinuity is smaller than that predicted by the model. This led to effective temperatures, as determined from the Paschen continuum, sometimes as much as several thousand degrees higher than those found from the Balmer jump for late B- or early A-type stars. With Hayes' calibration the Paschen continuum is steeper, giving a Balmer discontinuity about $0 . \mathrm{m} .13$ larger than Code's. Also, the steeper Paschen continuum implies a higher effective temperature, which requires models with smaller Balmer jumps for stars earlier than $\mathrm{AO}$ and helps to fit the discontinuity. Using this calibration, Wolff et al. (1968) have found that one can generally get good agreement between effective temperatures of A- and Btype stars found from the Paschen continuum and those found from the Balmer jump. Furthermore, the resulting temperature scale agrees well with that found by Hanbury Brown et al. (1967) from their interferometric observations. Earlier in this conference Oke and Schild (this volume, pp. 13-17) described their absolute calibration work at Palomar. Their preliminary results indicate substantial agreement with Hayes' calibration.

More work needs to be done on the absolute value of the flux from a star at a given wavelength. The value of $3.8 \times 10^{-9} \mathrm{ergs} \mathrm{cm}^{-2} \mathrm{sec}^{-1} \AA^{-1}$ at an effective wavelength of $5560 \AA$ for a star with $V=0^{\mathrm{m}} .0$ should be redetermined using a narrower bandpass than the approximately $100 \AA$ width interference filter used by Code and Whitford. We hope to remeasure this quantity at Wisconsin this fall, using as absolute standards both tungsten ribbon filament lamps calibrated by the National Bureau of Standards and the synchrotron radiation from the storage ring near Madison, which is capable of giving absolute as well as relative flux energy distributions. The present quality of spectrophotometric observations and model atmosphere predictions is such that the uncertainty of about $5-10 \%$ in the absolute flux value and the possible uncertainty in the effective wavelength at which it is measured is now just beginning to be felt.

Recently there have been several rediscussions of the effective temperature scale, especially for the earlier type stars. Table I compares these temperature scales. The first was given by Hanbury Brown et al. (1967) from their interferometric measurements. The second temperature scale shown is that of Wolff et al. (1968), who compared the Balmer line blanketed models of Mihalas with photometric observations using Hayes' recalibration of Vega. The third scale is that of Morton (1969), and Morton and Adams (1968), who identified stars with blanketed models through the observed UBV colors or the Balmer jump. The last column gives Heintze's (1969) temperature scale. He used line profiles as well as continuous energy distributions for its determination. Going towards earlier type stars the agreement among the four scales is quite good up to $(B-V) \approx-0.15$, where fairly large differences begin to appear. The 
TABLE I

Effective temperature scales

$\begin{array}{ccccc}(B-V) & \begin{array}{l}\text { Hanbury Brown } \\ \text { et al. }\end{array} & \text { Wolff et al. } & \begin{array}{l}\text { Morton and } \\ \text { Adams }\end{array} & \text { Heintze } \\ & & & & \\ -0.25 & & & & 25500 \\ -0.23 & 26600: & & 19700 & 20400 \\ -0.22 & 22900 & 18500 & 19100 & 19000 \\ -0.20 & 18600 & 14800 & 15100 & 16800 \\ -0.15 & 14250 & 12500 & 12500 & 13800 \\ -0.10 & 12400 & 11000 & 10400 & 12300 \\ -0.05 & 11150 & 10000 & 9600 & 9700 \\ 0.00 & 10250 & 9450 & 9150 & 9400 \\ +0.05 & 9600 & 8970 & 8780 & \\ +0.10 & 9100 & 8600 & 8430 & \\ +0.15 & 8650 & & 8120 & \\ +0.20 & 8250 & & 7850 & \\ +0.25 & 7850 & & 7600 & \\ +0.30 & 7500 & & 7000 & \\ +0.40 & 6850 & & 6420 & \\ +0.50 & 6325 & & 5920 & \\ +0.60 & 5875 & & & \end{array}$

uncertainties in the temperature determinations are greater for the hotter stars because the visual energy distributions are insensitive to effective temperature; ultraviolet observations are crucial here. The consistency of the scales determined in the visual is reassuring, but does not guarantee that this is the correct scale since all the models used in the temperature determinations could be incorrect in the ultraviolet. Nearly all the temperature scales may be systematically too hot for stars of earliest type, since model atmospheres with far-UV line blanketing were not generally used, and the effect of this blanketing is to lower $\theta_{\mathrm{e}}$ by a few hundredths, as noted earlier.

\section{Ultraviolet Observations}

Bless et al. (1968) reviewed the existing ultraviolet filter observations and compared them with model atmosphere calculations. We found that for some dozen stars observed by both Wisconsin and Boggess (1967) in the $2000 \AA-3000 \AA$ region, the agreement in the absolute fluxes was quite good, $\sim 0 \mathrm{~m} .25$. This probably represents a reasonable estimate of the accuracy attainable by unstabilized rocket photometry. Shortward of $2000 \AA$, however, the accuracy is often much poorer than this.

Observations of 35 stars at several wavelengths shortward and longward of $2000 \AA$, taken from various authors, were compared with models chosen according to their (B-V) colors and the Morton-Adams (1968) temperature scale, using blanketed models wherever possible. Most of the observations fell within $\pm 0^{\mathrm{m}} .5$ of the model predictions with more observations falling below the predicted flux level than above. This result may now be weakened somewhat since $\mathrm{OAO}$ and rocket observations suggest 
that blanketing may not be as great as predicted by the blanketed models. However, these models do not include the effects of weak lines. Flux measurements at $1427 \AA$ and $1115 \AA$ fell far below the model predictions, even with line-blanketed models. Since observations at $1376 \AA$ and $1314 \AA$ were in reasonable agreement with the models, it was suspected that $1427 \AA$ measurements and possibly the $1115 \AA$ measurements as well were in error rather than the model predictions. Recent observations by Carruthers (1969) have shown that the earlier NRL measurements at $1115 \AA$ should be increased by as much as an order of magnitude. I think a cautionary note is appropriate here. A few years ago there were thought to be very serious deficiencies in the observed UV fluxes below about $2500 \AA$, ranging up to factors of 10 or even 20 . Similarly at very, short wavelengths, around 1100 to $1400 \AA$, very large deficiencies were thought to exist. It now appears that the observations around $2500 \AA$ were biased by calibration problems and that the large deficiencies are not real. The same is at least partially true for the shorter wavelength observations. Changes in the temperature scale and the inclusion of some line blanketing in the models also improved the agreement. One must be fully aware of the difficulties of making accurate measurements in this wavelength region. Scattered light often affects the accuracy of laboratory calibrations and may result in an underestimate of observed fluxes. The sensitivity of some detectors decreases with time so that calibrations must be made as near to flight time as possible. Also, in some cases residual atmospheric extinction may not have been fully taken into account, again resulting in an underestimate of the real flux. Ultraviolet deficiencies (with respect to presently published models) may in fact exist, especially at short wavelengths for late B- to F-type stars; however, one must interpret the observations with caution.

Since the review by Bless et al. (1968) was written, a few other observations have been published. Yamashita (1968) observed half a dozen early-type stars in three spectral regions from 1060 to $1480 \AA$. In general, these observations appear to show flux deficiencies up to 1 to 2 magnitudes. However, the uncertainties in the measurements are very large, the smallest being quoted as 0.30 , the largest as $1^{\mathrm{m}} .3$. Thus it is not clear whether real deficiencies exist here. Stecher (1969a) has recently completed data reduction of observations obtained from several rocket flights. He flew an objective grating system which used the rocket roll to scan the spectrum with approximately $100 \AA$ resolution. About 25 stars were observed in this manner, ranging from an O5f star, $\zeta$ Pup, to an F5 IV star, $\alpha$ CMi. Stecher feels that his observations indicate considerable line blanketing in the 1800 to $2100 \AA$ range in hotter stars, and near $2300 \AA$ in the cooler stars. Stecher $(1969 \mathrm{~b})$ has also completed the reduction of data obtained with a $10 \AA$ resolution photoelectric spectrum scanner which he has flown on stabilized Aerobee rockets. These observations cover the spectral range from $3000 \AA$ to Lyman $\alpha$, and contain a wealth of information on the stronger spectral lines, as well as on the continuous energy distribution. Opal et al. (1968) have observed $\eta$ UMa with an objective prism telescope over the wavelength region 1175 to $1800 \AA$. The shape of the energy distribution agrees nicely with the model atmosphere predictions. However, the absolute flux is lower than predicted by slightly less than a factor of 2 . 
This is just within the quoted calibration error and it seems likely to me that the discrepancy is caused by calibration difficulties rather than by an unknown opacity source, especially since this unknown absorber would have to be grey over the spectral region 1175 to $1800 \AA$.

Additional ultraviolet observations have been made by Henize et al. (1968) in the wavelength region 2300 to $5000 \AA$, with instrumentation carried on the Gemini 10 , 11 , and 12 spacecrafts. They used a camera with a $30^{\circ}$ field of view to which was attached either an objective prism or an objective grating. The former gives a dispersion of $1400 \AA$ per $\mathrm{mm}$ at $2500 \AA$, while the latter gives a dispersion of $184 \AA$ per $\mathrm{mm}$. Several hundred stars have been observed in the Orion and Scorpius regions with the objective prism system. Henize is currently reducing observations of about 15 stars taken with the grating system in Scorpius and Orion. The group at Geneva, under the direction of Golay, is continuing its program of balloon observations with an objective prism camera. Recently they had a successful flight at an altitude of about 40 kilometers. They have recorded the spectra of many stars down to about $2500 \AA$ with resolution as high as $50 \AA$ and are now reducing this large amount of data. Filter photometry in the 2000 to $3000 \AA$ region and $100 \AA$ resolution spectrophotometry have also been carried out by Campbell, Sudbury, and their colleagues at Edinburgh, who flew small telescopes on sounding rockets. These observations are also in the reduction stage.

Later we will hear about additional ultraviolet photometry being done by Soviet, U.S., and French experimenters.

Finally, I would like to say a little about OAO observations. In the paper on interstellar extinction we mentioned one result of wider interest, namely, that unreddened early-type stars of the same visual spectral type have the same continuous spectrum in

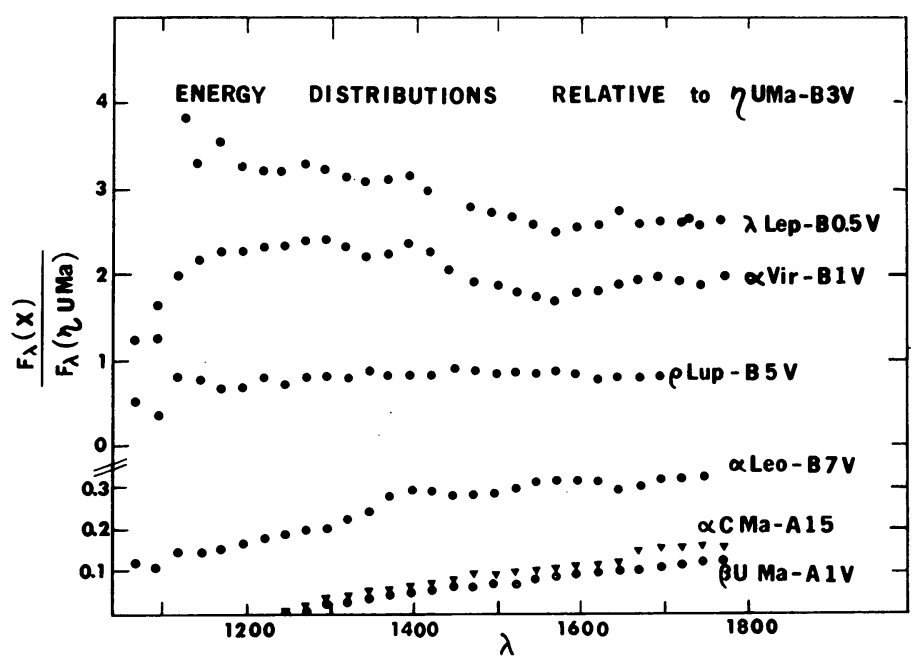

Fig. 2. Preliminary relative energy distributions in the far ultraviolet derived from Orbiting Astronomical Observatory scans. 
the ultraviolet. If this is found to be generally true it will greatly simplify the interpretation and analysis of stellar spectra.

Figure 2 displays preliminary scanner data of seven stars from B0.5 V to AlV, normalized to $\eta \mathrm{UMa}$, a B3 V star, which was assumed to have $T_{\mathrm{e}}=17800 \mathrm{~K}$. The observations were compared with blanketed models with $\log g=4.0$. Effective temperatures so determined for the B5 and earlier stars are a little hotter by 1000-2000 K than those given by Morton and Adams (1968). Also, the observed fluxes from the cooler stars are less than those predicted by the models by $0^{\mathrm{m}} .5$ to $1^{\mathrm{m}} .0$. Possibly the inclusion of carbon opacity in the models would decrease this apparent discrepancy. Notice particularly the scan of Sirius in Figure 2. A noteworthy feature of this scan is the distinct discontinuity at about $1680 \AA$ although none appears at $1520 \AA$. The former wavelength corresponds to photoionization from the ${ }^{1} D$ level of silicon, about one volt above the ${ }^{3} P$ ground state, whose absorption edge lies at $1520 \AA$. If the jump at $1680 \AA$ is real it is difficult to understand the absence of a discontinuity corresponding to ionization from the ground state.

In addition to the two spectrum scanners mentioned in the extinction paper, the Wisconsin OAO package also includes four telescopes of $20 \mathrm{~cm}$ aperture for filter photometry in nine different wavelength bands from Lyman $\alpha$ to the blue. The filter half-widths are about $300 \AA$. Typically our limit for early-type stars is about $7^{\mathrm{m}}$ visual, although we have gone considerably fainter. This limit is set primarily by the confusion of other stars in the field for the 10' field stop we use.

With these photometers we have so far observed about 450 stars having a wide range of spectral type, luminosity class, reddening, and peculiarities. As with the spectral scans, the photometric quality is quite good. Figure 3 shows photometry at

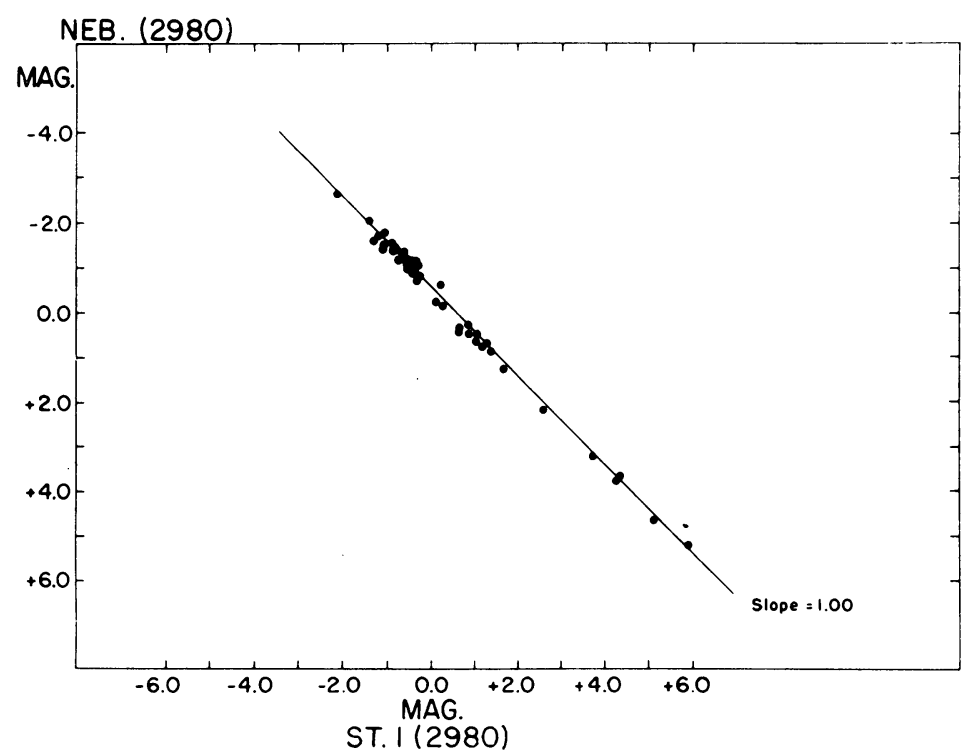

Fig. 3. $2980 \AA$ filter photometry of the same stars with two different OAO instruments. 
$2980 \AA$ done simultaneously with two different instruments over a period of several weeks. These instruments have nearly identical bandpasses. Over the $7^{\mathrm{m}}$ range of the observations, the average deviation of an observation is $0^{\mathrm{m}} .02-0^{\mathrm{m}} .03$.

Soon, hopefully, already existing OAO data will be put in a form suitable for machine reduction so that the various small corrections can be made where necessary. This large amount of homogeneous data for many stars should enable us to begin answering some of the questions raised here.

\section{References}

Auer, L. A. and Mihalas, D.: 1969, Astrophys. J. 156, 681.

Bahner, K.: 1963, Astrophys. J.138, 1314.

Bless, R. C., Code, A. D., and Houck, T. E.: 1968, Astrophys. J. 153, 561.

Boggess, A.: 1967, private communication.

Brown, R. Hanbury, Davis, J., Allen, L. R., and Rome, J. M.: 1967, Monthly Notices Roy. Astron. Soc. 137, 393.

Carruthers, G. R.: 1969, Astrophys. Space Sci. 5, 387.

Code, A. D.: 1960, in Stars and Stellar Systems, Vol. VI, Stellar Atmospheres (ed by J. L. Greenstein), University of Chicago Press, Chicago, Chapter 2, p. 50.

Elst, E. W.: 1967, Bull. Astron. Inst. Netherl. 19, 90.

Griem, H. R.: 1960, Astrophys. J. 132, 883.

Griem, H. R.: 1962, Astrophys. J. 136, 422.

Hayes, D.: 1967, unpublished dissertation, University of Calif., Los Angeles.

Heintze, J. R. W.: 1969, Bull. Astron. Inst. Netherl. 20, 154.

Henize, K. G., Wray, J. D., and Wackerling, L. R.: 1968, Bull. Astron. Inst. Csl. 19, 279.

Hickok, F. R. and Morton, D. C.: 1968, Astrophys. J. 152, 203.

Klinglesmith, D. A. and Fischel, D.: 1969, private communication.

Mihalas, D.: 1964, Astrophys. J. Suppl. Ser. 9, 321.

Mihalas, D.: 1965, Astrophys. J. 141, 564.

Mihalas, D.: 1966, Astrophys. J. Suppl. Ser. 13, 1.

Mihalas, D. and Morton, D. C.: 1965, Astrophys. J. 142, 253.

Morton, D. C.: 1969, Astrophys. J., 158, 629.

Morton, D. C. and Adams, T. F.: 1968, Astrophys. J. 151, 611.

Oke, J. B.: 1964, Astrophys. J. 140, 689.

Opal, C. B., Moos, H. W., Fastie, W. G., Bottema, M., and Henry, R. C.: 1968, Astrophys. J. 153, L179.

Stecher, T. P.: 1969a, Astrophys. J. 74, 98.

Stecher, T. P.: 1969b Astrophys. J., in press.

Strom, S. E. and Avrett, E. H.: 1965, Astrophys. J. Suppl. Ser. 12, 1.

Underhill, A. B.: 1963, Publ. Dom. Astrophys. Obs. Victoria 11, 467.

Wolff, S. C., Kuhi, L. V., and Hayes, D.: 1968, Astrophys. J. 152, 871.

Yamashita, K.: 1968, Astrophys. Space Sci. $2,4$.

\section{Discussion}

Morton: The non-LTE effects which may reduce the Lyman continuum appear to decrease with increasing effective temperature so that we have ignored this effect for the hottest models.

Bless: Two models have so far been published, the hotter one just getting into the $T_{\mathrm{e}}$ range you are interested in. It would be nice to have an even hotter model in order to check this point.

Underhill: Feautrier has constructed a few model atmospheres taking fully into account the effects of non-LTE in the hydrogen spectrum. The result is a rise in the temperature in the outermost part of the atmosphere. This hot plateau is more significant for type A0 than for B0, as I recall, and it may influence the formation of strong lines. 
Bless: I think Auer and Mihalas found the same effect.

Morton: I suspect that non-LTE effects will be important in the cores of the lines, but not in the damping wings which are formed deep in the atmosphere and are the main contribution to the blanketing.

Underhill: The rather large differences in fluxes near $2000 \AA$ predicted from A type models when different sources of opacity are used arise chiefly because at this wavelength one is far in the shortward wing of the black body curve for the relevant temperatures. Consequently a change in temperature of a few hundred degrees causes a large percentage change in the small flux emitted. To interpret the absolute fluxes in the region accurately one would need to be very sure of the temperature of the atmosphere.

Gingerich: In the process of computing an extensive grid of model stellar atmospheres (to be published in the Proceedings of the Third Harvard Smithsonian Stellar Atmosphere Conference), D. Carbon has investigated the observational effects of a mixing-length theory of convection. These models include opacity from silicon, carbon, and schematic line-blocking. The convective effects are greatest for a main-sequence star with $T_{\mathrm{eff}}=7500^{\circ}$, and appear in the Balmer discontinuity as well as at $1683^{+} \AA$. The effects are small in the UV, and occur in a region of uncertain opacity. They are essentially independent of the mixing-length chosen. We conclude that UV observations will not be able to distinguish between various amounts of convection.

Henize: Have you observed the predicted flux differences between pole-on and equator-on rapidly rotating stars?

Bless: We have filter photometry observations of rapidly rotating stars, but have not yet reduced them.

Stecher: The star $\beta$ Lup B5 V looked the same as $\eta$ UMa B3 V. Is this a property of rotation or is it some other effect such as a classification error?

Bless: I don't think it has to dn with rotation, nor do I know how certain the classification is. 